\title{
Diversidad fitoplanctónica en los cuencos principal y norte de la laguna Don Tomás (La Pampa. Argentina)
}

\author{
Susana Beatriz Álvarez ${ }^{1, *}$, Graciela Inés Bazán ${ }^{1}$ y Jaime Nicolás Bernardos ${ }^{1,2}$ \\ ${ }^{1}$ Facultad de Ciencias Exactas y Naturales, Universidad Nacional de La Pampa. Uruguay 151, Santa Rosa (6300), \\ La Pampa, Argentina. gibazan@exactas.unlpam.edu.ar \\ ${ }^{2}$ INTA, Estación Experimental Agropecuaria Guillermo Covas, Anguil, La Pampa, Argentina. Ruta 5 km 580.cc. \\ jbernardos@anguil.inta.gov.ar
}

* Autor responsable de la correspondencia: sbalvarez@exactas.unlpam.edu.ar

Recibido: 14/5/07

Aceptado: 27/7/09

\begin{abstract}
Phytoplanktonic diversity in the main and north basins of the Don Tomas lagoon (La Pampa. Argentina)

This work analyzes the composition, diversity and specific richness of phytoplankton species in the Don Tomás lagoon, a natural shallow lake which belongs to the Eastern physiographic region of the La Pampa province, during an annual cycle (2002-2003). The lake has an area of about 200 ha, 2, 5-3 m in depth, and in this opportunity two sampling areas are considered, the main and north basins. There were no temperature differences between both basins $(p>0.5)$ and the variation range between winter and summer was between $11^{\circ}$ and $26^{\circ} \mathrm{C}$. Significant differences were found between basins regarding $\mathrm{pH}(p<0.01)$ Phytoplankton was characterized by an extensive variety of taxa qualitatively dominated by green algae. The specific richness of the Main Basin was larger than for the North Basin. The Main Basin showed a sharp decline in the specific richness in November associated with a blooming of Cyanophyceae. This blooming occurred again between March and April 2003 with a dominance of Planktothrix agardhil (Gom.) Anag. Kom., species that produced a high fish mortality.
\end{abstract}

Key words: Phycoflora, diversity, seasonal distribution.

\section{RESUMEN}

\section{Diversidad fitoplanctónica en los cuencos principal y norte de la laguna Don Tomás (La Pampa. Argentina)}

El presente trabajo analiza la composición, diversidad y riqueza específica de las especies fitoplanctónicas de la Laguna Don Tomás, un lago somero natural que pertenece a la región fisiográfica oriental de la provincia de La Pampa, durante un ciclo anual (2002-2003). La laguna posee una superficie aproximada de 200 ha, con una profundidad de 2,5-3 m y en esta oportunidad se consideran dos áreas de muestreo, los cuencos Principal y Norte. No se hallaron diferencias en los valores de temperaturas de ambos cuerpos de agua ( $\mathrm{p}>0.5)$ y el rango de variación entre invierno y verano fue entre $11^{\circ}$ y $26^{\circ} \mathrm{C}$. Con respecto al pH se hallaron diferencias significativas $(\mathrm{p}<0.01)$. El fitoplancton estuvo caracterizado por una amplia variedad de taxa cualitativamente dominada por algas verdes. La riqueza específica en el Cuenco Principal fue mayor que en el Cuenco Norte. El Cuenco Principal evidenció un brusco descenso de la riqueza específica en el mes de noviembre asociado a una floración de cianofíceas, reiterada entre marzo y abril de 2003, con una floración de Planktothrix agardhii (Gom.) Anag. Kom., la cual ocasionó una elevada mortandad de peces.

Palabras clave: Ficoflora, diversidad, distribución estacional. 


\section{INTRODUCCIÓN}

Existen numerosos aportes en relación al estudio de lagos someros (lagunas), tanto en zonas tropicales y subtropicales como en zonas templadas, los que están relacionados con las regímenes hidrológicos de ríos (Rai, H. \& G. Hill, 1980; García de Emiliani \& Depetris, 1982; Zolacar de Domitrovic et al., 1982; Boltovskoy et al., 1990; Izaguirre \& Vinocur, 1994).

Las lagunas de la pampa deprimida de la provincia de Buenos Aires) registran un estado trófico que varía entre eutrófico e hipertrófico (Quirós et al., 2002), al igual que las provincia de La Pampa, pero a diferencia de éstas la mayor parte de ellas carecen de relación con regímenes hidrológicos, salvo las lagunas de la cuenca del río Desagüadero-Salado-ChalideuvuCuracó (Lag. La Dulce y Lag. Urrelauquen, Departamento Curacó) (Quirós R., 1988).

Los trabajos ficológicos de tipo taxonómico comenzaron a realizarse en la provincia de La Pampa, en la década del noventa (Álvarez, 2002; Álvarez et al., 1998a; 1998b; 2004; 2005; Bazán et al., 1996; 1998; 2004; Wenzel et al., 1996; Romero 1993; 1995; Maidana \& Romero, 1995).

La laguna Don Tomás es un cuenco natural semipermanente que se utilizó como estanque receptor de los líquidos cloacales de la ciudad de Santa Rosa. A partir del año 1991 esta evacuación tiene lugar por medio de un sistema de bombeo mixto, por entubación, hasta el Parque Industrial y, a partir de allí, se desviaron las aguas a través de un canal a cielo abierto hacia el Bajo de Giuliani. La laguna Don Tomás se transformó en un cuerpo de agua utilizado con fines recreativos, cuyo exceso de agua es bombeado también hacia el cuenco mencionado en meses y años de fuertes precipitaciones (Carballo et al., 2000).

Asimismo se han realizados en la misma trabajos de tipo taxonómico en estudios florísticos de las Div. Chlorophyta (Álvarez, 1992) y Div. Cyanophyta (Álvarez et al., 1994) de la provincia. En los mismos se registraron 40 taxa de la Div. Chlorophyta y 20 de la Div. Cyanophyta.

El presente trabajo tiene como objetivo analizar y comparar la composición, diversidad, riqueza específica y la distribución estacional de las especies fitoplanctónicas en los Cuencos Principal y Norte de La Laguna Don Tomás, durante un ciclo anual.

\section{ÁREA DE ESTUDIO}

\section{Descripción e historia de la Laguna Don Tomás}

La Laguna Don Tomás, situada en la región occidental de la ciudad de Santa Rosa. Capital de la Provincia de La Pampa, Argentina, pertenece al grupo de las lagunas de la región fisiográfica oriental de la provincia y posee una superficie aproximada de 200-220 ha, con una profundidad media que oscila entre 2.5 y 3 metros. La temperatura media anual para la ciudad de Santa Rosa es de $15^{\circ} \mathrm{C}$ y la Precipitación Media Anual de $600 \mathrm{~mm}$ (Servicio Meteorológico Nacional).

El área de estudio se encuentra ubicada en la región Neotropical, Dominio chaqueño, Provincia biogeográfica pampeana, Distrito fitogeográfico pampeano-occidental (Cabrera y Willink, 1980).

La Laguna "Don Tomás", es una depresión natural localizada en $36^{\circ} 37^{\prime} 30.2^{\prime \prime}$ S y $64^{\circ} 18^{\prime} 29.8^{\prime \prime} \mathrm{W}$ (Fig. 1) elongada en el sentido Nor-Noroeste y Este-Sureste, y se halla circundada por una suave pendiente meridional de $1.5 \%$ de gradiente y una septentrional de inclinación mayor, aproximadamente $5 \%$, ambas de configuración convexa.

El lugar fue utilizado para fabricación de ladrillos hasta la década del sesenta, iniciándose de

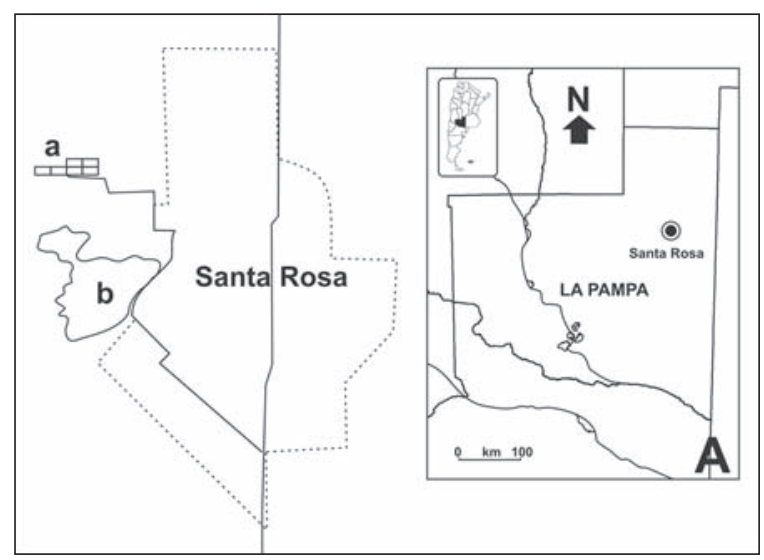

Figura 1. Localización de la laguna Don Tomás. Don Tomás Lake location. 
esa forma la actividad antrópica, con el consecuente impacto posterior. El agua acumulada en esta depresión provocó un aumento en su dimensión natural mediante el aporte de líquidos cloacales de la ciudad y los del molino harinero, y el agua de origen pluvial proveniente de la ciudad de Santa Rosa. Estos nuevos aportes conformaron una laguna de tipo semipermanente, fuertemente contaminada debido a que los mecanismos de autodepuración no llegaron a funcionar eficazmente. Estas circunstancias originaron una creciente eutrofización natural, de tipo cultural, es decir, ocasionada por una aceleración antropogénica (Laws, 1993).

Este bajo salino endorreico presenta un régimen de alimentación constituido por el aporte de las aguas precipitadas sobre su superficie, el escurrimiento pluvial de la ciudad de Santa Rosa y la carga y descarga de la napa freática. Existe un cuenco donde se desarrollan actividades recreativas y al que nosotros hemos denominado "Cuenco Principal" y en la zona aledaña nos referiremos al "Cuenco Norte". En la actualidad el sistema de alcantarillado sanitario es independiente del sistema de drenaje pluvial.

\section{MATERIAL Y MÉTODOS}

Para el presente estudio se definieron seis estaciones de muestreo en área perimetral de la laguna, cinco en el Cuenco Principal y una en el Cuenco Norte. Los puntos seleccionados en el Cuenco Principal fueron: 1) Zona de juncos, 2) Puente del embarcadero, 2) La Cruz, 3) El Faro, 4) Alcantarilla desagüe Av. Perón y la estación 6) frente a la cruz, en el Cuenco Norte. El muestreo se realizó con una frecuencia mensual, durante el período comprendido entre agosto de 2002 y julio del 2003.

Las muestras cualitativas de fitoplancton se obtuvieron por arrastre con una red de $20 \mu \mathrm{m}$.

El fitoplancton se observó en vivo bajo microscopio Kyowa Medilux para realizar una correcta observación de formas coloniales, agregados celulares e individuos flagelados. Las muestras se fijaron con formaldehído al $4 \%$ y se depositaron en el herbario de la Facultad de Agronomía de la UNLPam, bajo las siglas SRFA legado Alvarez-Bazán.
Las determinaciones específicas se realizaron siguiendo Geitler, 1932; Komárek \& Agnostidis, 1989; 1999; 2005; Komárek \& Fott, 1983; Prescott, 1951.

Simultáneamente a la extracción de muestras, se registraron parámetros físico-químicos tales como: temperatura del agua y del aire, la conductividad se registró con un conductímetro LUFMAN Mod. HIDROSALT 12 y el pH, con un pHmetro MATAROM PHMETER E-516. Mediante el Disco de Secchi, se obtuvo la transparencia y profundidad.

Se confeccionó una tabla de presencia-ausencia de los taxa y su frecuencia relativa según $F_{r}=$ $S_{i} / N \cdot 100$. Donde $S_{i}$ es presencia de la especie, $i$ es de inventarios y $N$ es el número total de inventarios.

Se calculó la riqueza específica mensual para cada cuenco. Se formaron grupos de especies por medio de análisis de agrupamientos por el método Ward y el complemento de la distancia binaria, utilizándose el software $\mathrm{R}$ versión 2.9.

\section{RESULTADOS}

\section{Aspectos Ambientales}

La transparencia del agua osciló entre 0.07 y $0.28 \mathrm{~m}$ de profundidad del disco de Secchi, no

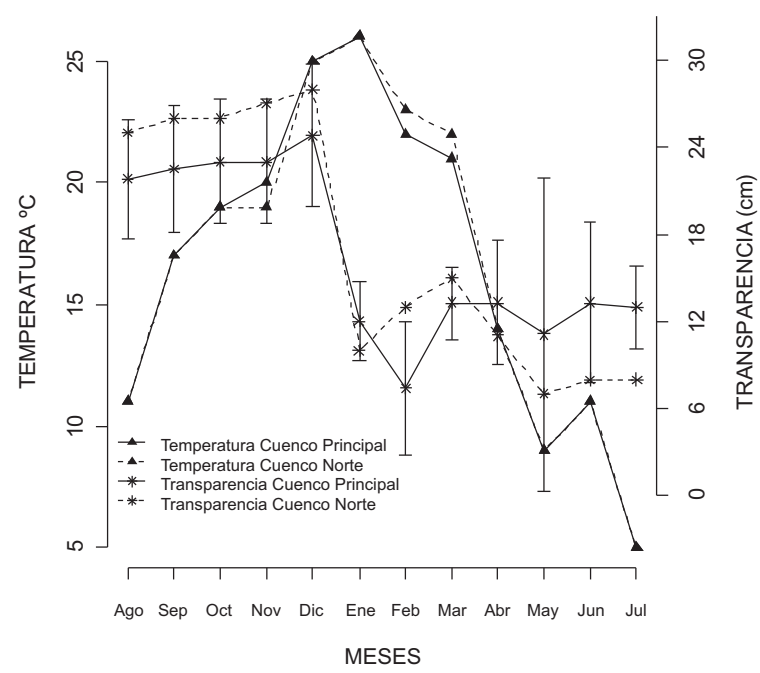

Figura 2. Transparencia y temperatura del agua durante el ciclo anual 2002-2003 en los Cuencos Principal y Norte del Sistema de la laguna Don Tomás. Water transparency and temperature during the annual cycle 2002-2003 for the Main and North basins of Don Tomás Lake. 
encontrándose diferencias significativas entre ambos cuencos ( $p>0.64)$ (Fig. 2). La transparencia mínima en el Cuenco Principal se halló en el mes de febrero, en concordancia con el verano en el hemisferio Sur, no obstante, es de destacar la importante variabilidad hallada entre sitios de muestreo.

La temperatura del agua de ambos cuencos ha sido similar, entre un máximo y un mínimo de 26 y $5{ }^{\circ}$ C. $(p>0.58)$ (Fig. 2$)$.

La conductividad varió entre 1400 y $1950 \mu$ $\mathrm{S} \mathrm{cm}^{-1}$, para ambos cuencos, no hallándose diferencias significativas. El pH fluctuó entre 6 y 8.3 para el cuenco Principal y entre 8.5 y 11 para el cuenco Norte, (Fig. 3) presentado diferencias de medias altamente significativas $(p<0.01)$.

\section{Análisis de cualitativo}

La riqueza específica fitoplanctónica a lo largo del año en el Cuenco Principal fue de 146 taxones y la del Cuenco Norte de 110 taxones.

Las divisiones estudiadas estuvieron representadas para el Cuenco Principal por la Div. Chlorophyta (49\%), Div. Cyanophyta (30\%), Div. Bacillariophyta (16\%) y para otras algas el

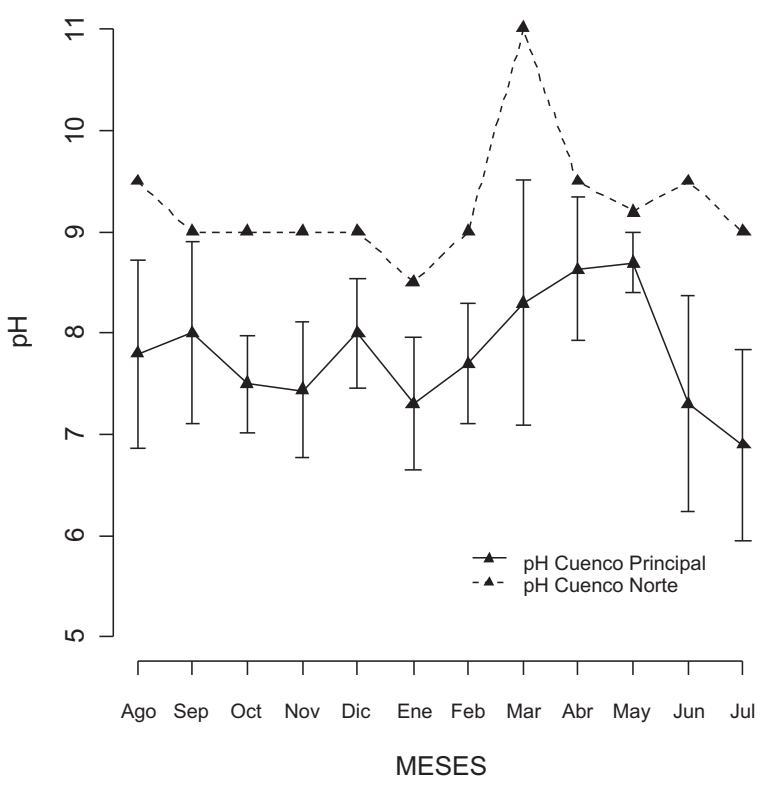

Figura 3. pH del agua durante el ciclo anual 2002-2003 en los Cuencos Principal y Norte del Sistema de Lagunas Don Tomás. Water pH during the annual cycle of 2002-2003 for the Main and North basins of Don Tomás Lake.

$5 \%$ (Div. Euglenophyta, Chrysophyta, Dinophyta y Cryptophyta) (Fig. 4).

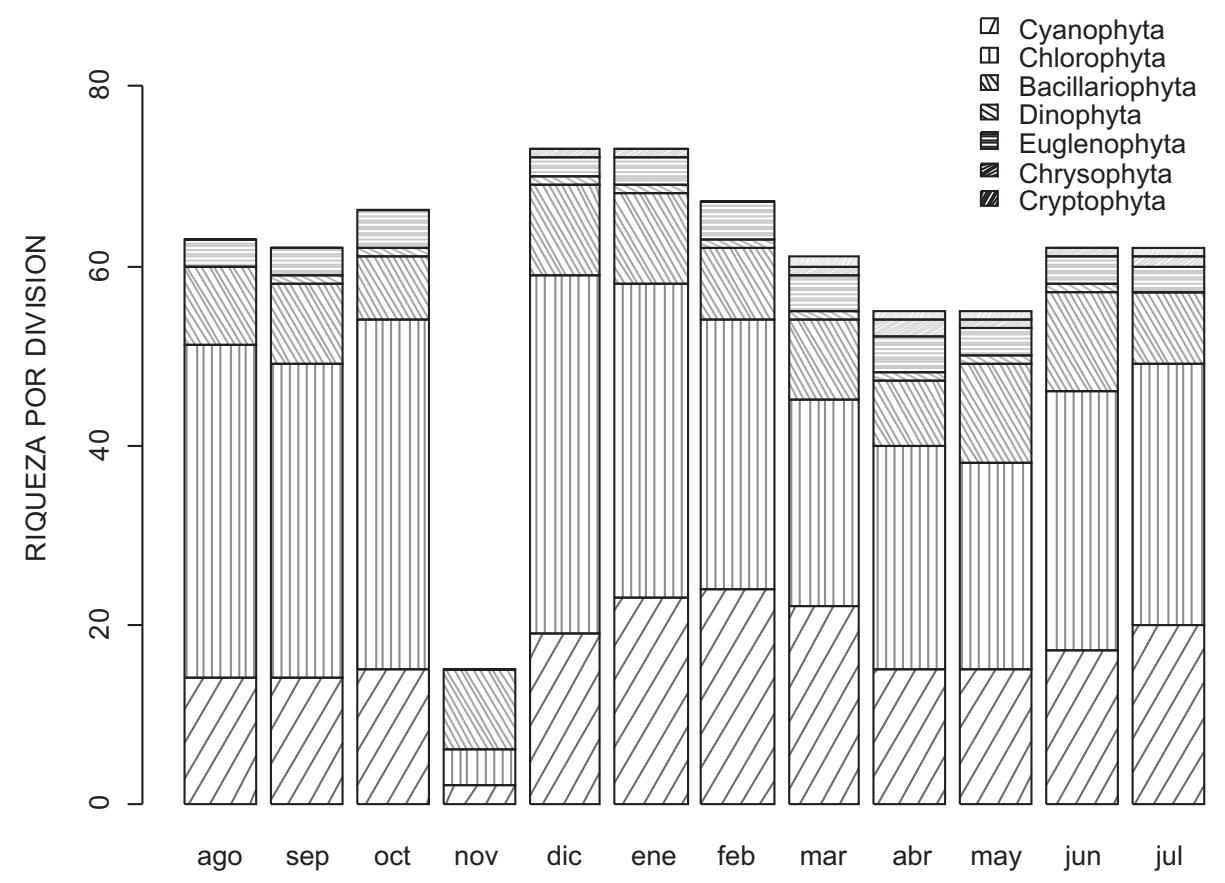

Figura 4. Frecuencia de especies por División a lo largo del ciclo anual anual 2002-2003 en el Cuenco Principal del Sistema de Lagunas Don Tomás. Species Frecuency (by Division) during the annual cycle 2002-2003 for the Main basin of Don Tomás Lake. 


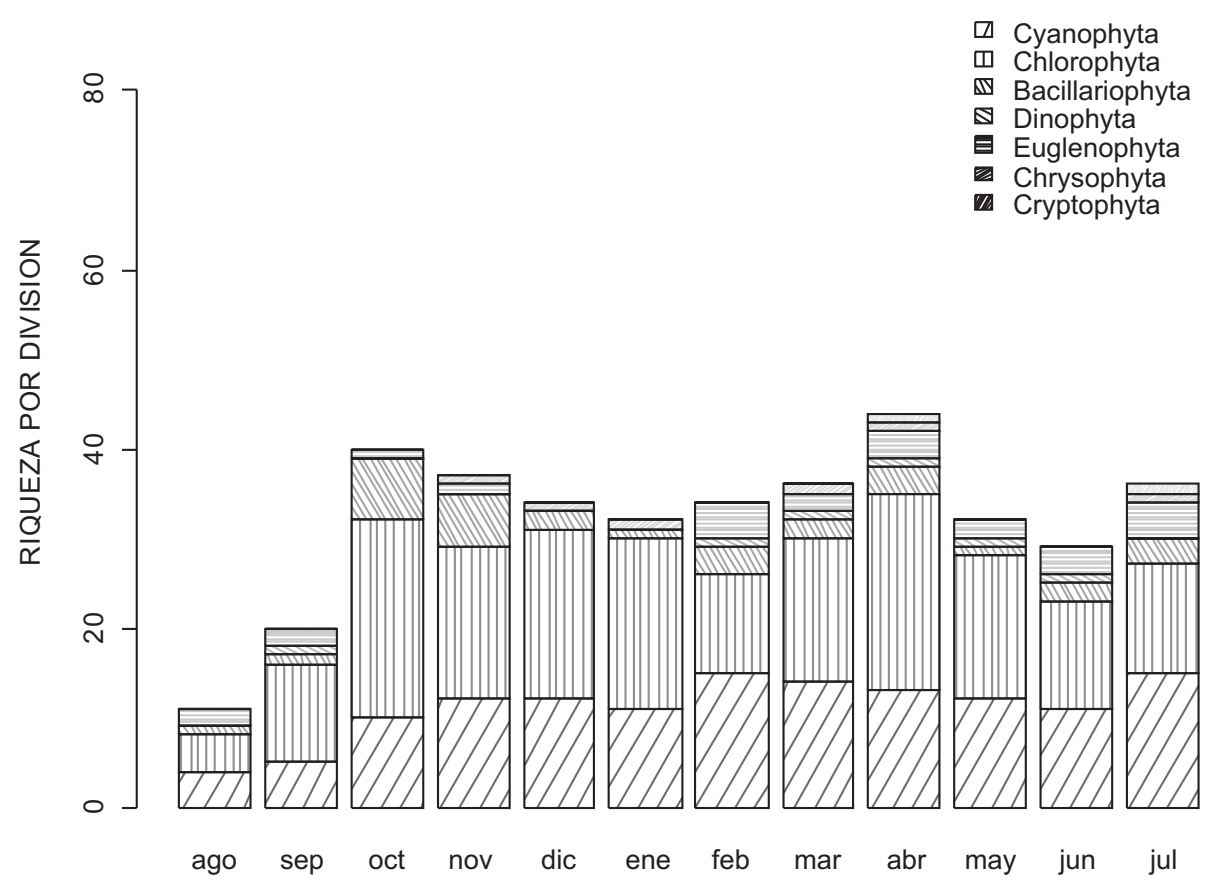

Figura 5. Frecuencia de especies por División a lo largo del ciclo anual anual 2002-2003 en el Cuenco Norte del Sistema de Lagunas Don Tomás. Species frequency (by Division) during the annual cycle of 2002-2003 for the North basin of Don Tomas.

El Cuenco Norte tuvo una composición de $49 \%$ para la Div. Chlorophyta, $34 \%$ para Cyanophyta; $10 \%$ para Bacillariophyta y el $7 \%$ restante para individuos incluidos en las divisiones Euglenophyta, Chrysophyta, Dinophyta y Cryptophyta (Fig. 5).

La riqueza específica se presentó constante en ambos cuencos salvo en el mes de noviembre en el cuerpo principal en el que se evidenció una brusca baja de la misma asociada a una floración de cianofíceas.

Para el Cuenco Principal en cuanto al número de especies por División, la Div. Chlorophyta se encontró en mayor número durante todos los meses, seguida de la Div. Cyanophyta, y en menor proporción por las Div. Heterokontophyta, Clase Bacillariophyceae.

En el Cuenco Norte, el predominio de especies de Chlorophyta sobre Cyanophyta fue marcado. Durante los meses de febrero y julio se observó una mayor riqueza específica de Cyanophyta.

Asociado a las floraciones de cianofíceas la Clase Bacillariophyceae predominó en ambos cuencos en el mes de noviembre. El $23 \%$ de las especies se hallaron presentes en nueve de los meses estudiados, destacándose Anabaenopsis arnoldii, Planthotrix agardii, Phomidium sp, Chlorella elipsoidea, Monoraphidium grifithii, $M$. arcuatum, Tetraedrum minimun, Actinastrum raphidioides, Scenedesmus acuminatus, Aulacoseira sp., Cyclotella sp., Navicula sp., Euglena sp.1.

En el cuenco principal, 30 de 146 especies, se hallaron en al menos 9 meses del año, mientras que en Cuenco Norte se encontraron presentes sólo 7 de 110 especies, entre ellas Aphanothece stagnina, Merismopedia tenuisima, Microcystis flos-aquae, Chamaesiphon subglobosum, Tetraedron minimum, Scenedesmus acuminatus, Euglena sp.

A lo largo del ciclo anual se encuentra que los meses de diciembre y enero fueron los que presentaron la mayor riqueza específica (73) para el Cuenco Principal, mientras que el mes de abril fue el que presentó la mayor riqueza específica (44) en el Cuenco Norte. En ambos cuencos el mayor número de especies pertenecieron a la División Chlorophyta (Figs. 4 y 5).

El análisis de agrupamientos muestra 5 grupos de asociaciones algales bien definidos, tan- 
to en Cuenco Principal como en el Cuenco Norte. El Cuenco Principal presentó un grupo de algas conformado por Microcystis sp, Coelomorum sp, Nostoc commune, Oscillatoria prolifica, Pandorina sp, Pediastrum boryanum var boryanum, Francecia ovalis, Scenedesmus opoliensis var carinatus y un segundo grupo integrado por Cyanocystis sp, Gomphophaeria lacustris, Scenedesmus quadrispina, Stephanodiscus sp y Actinodiscus sp., como los más representativos. A su vez, el Cuenco Norte presentó la asociación de Alaucoseira sp, Euastrum, Tetrastrum staurogeniaforme, Gomphophaeria lacustres y Tetraedron caudatum, con un marcado un predominio de Chlorococcales y la presencia de Desmidiaceae junto a una diatomea. En la segunda asociación hay una fuerte presencia de cianofíceas como Coelomorum sp., Oscillatoria acuta, Oscillatoria okeni, y Chloroccocales (Pediastrum sp, Crucigenia sp.) y Melosira sp.

\section{DISCUSIÓN Y CONCLUSIONES}

El fitoplancton del sistema Laguna Don Tomás está caracterizado por una amplia variedad de taxa, cualitativamente dominada por algas verdes. El $48-49 \%$ del total de la ficoflora pertenece a las Chlorophyceae, preferentemente del grupo de las Chlorococcales. La composición del fitoplancton del sistema muestra similitudes con la de aquellas lagunas estudiadas en la provincia Pampa (Álvarez, 2002; Álvarez et al., 1998a; 1998b; 2004; 2005; Bazán et al., 1996; 1998; 2004; Wenzel M.T et al., 1996; Romero 1993; 1995; Maidana \& Romero, 1995).

El número de taxa registrados en la actualidad fue mayor que en los estudios florísticos de las Div. Chlorophyta (Álvarez, 1992) y Cyanohyta (Álvarez et al., 1994) de la provincia. En los mismos se registraron 40 taxa de la Div. Chlorophyta y 20 de la Div. Cyanophyta, en el Sistema de la Lag. Don Tomás.

La sucesión estacional y la composición específica del fitoplancton fueron similares en las diferentes estaciones de muestreo durante el estudio. Las razones de estas similitudes puede de- berse a que las estaciones tuvieron características físicas y químicas muy parecidas.

Las diatomeas pennadas predominaron en ambos cuencos.

Planthotrix aghardii fue la especie dominante en el crecimiento masivo registrado en el cuenco Norte, siendo la especie toxicogénica más frecuente en ambos cuencos (Marshall et al. 2008).

La variación estacional del fitoplancton está relacionada con las fluctuación de los factores ambientales (Vila \& Pardo, 2003) tales como pH, turbidez, temperatura y la transparencia en primer lugar.

La temperatura del agua regula fuertemente las variaciones estacionales de fitoplancton (Richardson et al., 2000). El aumento de biomasa algal durante primavera y verano en el sistema de la Laguna Don Tomás, podría ser el resultado del aumento de la temperatura del agua.

La luz recibida es el mayor recurso utilizado por el fitoplancton y determina un patrón de variabilidad espacial y temporal (Litchman, 2000). La inexistencia de una arboleda rodeando la laguna Don Tomás, permite la penetración de una intensa radiación solar sobre la misma, en concomitancia con un aumento de la autotrofia.

El rango mínimo de transparencia en el cuenco principal se observó en los meses de febrero y mayo de 2003, con la floración de Planktothrix agardii (Gom.) Anagh. \& Kom. coincidente con una elevada mortandad de peces.

En los lagos naturales los niveles de turbidez son menores que en los lagos urbanos artificiales, la combinación de alta concentración de algas y turbidez reduce la transparencia del agua, los niveles de turbidez están asociados a la lixiviación de los sedimentos contiguos (disueltos y sedimentables) durante la época de lluvia (Schueler \& Simpson, 2003).

El cuenco Norte, presenta una disminución en su transparencia debido a la falta de aportes de agua produciéndose una elevada concentración de biomasa a partir de enero de 2003, situación que aún persiste. Estas algas, preferentemente del grupo de las cianofíceas, que no son pastoreadas por herbívoros, producen efectos organolépticos no deseados en el entorno del cuenco (Laws 1993). 


\section{AGRADECIMIENTO}

A la Dra. Ana Luján Martinez de Fabricius por la lectura crítica del manuscrito.

\section{REFERENCIAS}

ÁLVAREZ, S. B. 1992. Algas de aguas continentales de La Pampa. Argentina. I. O.Volvocales y O.Chloroococcales. Rev. Fac. Agronomía. UNLPam., 6(2): 35-51.

ÁLVAREZ, S. B. 2002. Estudio bioecológico y citotaxonómico del fitoplancton de una laguna eutrofizada, reservorio final de las aguas residuales de la ciudad de Santa Rosa, La Pampa. Tesis Universidad Nacional del Sur. Bahía Blanca. Argentina. 272 pp.

ÁLVAREZ, S. B. \& G. I. BAZÁN.1994. Cianofíceas Continentales de Pcia. de La Pampa. (Argentina). Rev. Fac. Agronomía. UNLPam., 7(2): 43-62.

ÁLVAREZ, S. B., G. I. BAZÁN \& M. T. WENZEL. 1998a. Blue green algae of Chroococcales and Chamaesiphonales orders in intermitent streams and isolated stagnant pools within the Lihuel Calel National Park (La Pampa province) in Argentina. Acta Hydrobiologica, 40: 131-146.

ÁLVAREZ, S. B., G. I. BAZÁN \& M. T. WENZEL 1998b. Blue green algae of Hormogonales orders (Nostocaceae, Scytonematacae, Microchaetaceae Families) in intermitent streams and isolated stagnant pools within the Lihuel Calel National Park (La Pampa province) in Argentina. Acta Hydrobiologica, 40: 147-160.

ÁlVAREZ, S. B., G. I. BAZÁN \& E. R. PARODI. 2004. Ficoflora del Reservorio final de aguas residuales de Santa Rosa ( La Pampa, Argentina). Cyanophyta y Chlorophyta (Tetraselmidales, Volvocales y Chlorococcales). Bol. Soc. Argent. Bot., 39(1-2): 277-293.

ÁLVAREZ, S. B., A. E. BIASOTTI, J. BERNARDOS \&G. I BAZÁN. 2005 . Ficoflora de la laguna Don Tomás (La Pampa, Argentina). Junio 2005. Biología Acuática, 22: 1-6.

BAZÁN, G. I., M. T. WENZEL \& S. B. ÁLVAREZ. 1996. Cianoficeas nuevas para Argentina del Parque Nacional Lihuel Calel (Pcia. de La Pampa). Darwiniana. 34(1-4): 285-292.

BAZÁN, G. I., S. B. ÁLVAREZ \& M. T. WENZEL. 1998. Blue green algae of Oscillatoriaceae Family in intermitent streams and isolated stagnant pools within the Lihuel Calel National Park (La Pampa province) in Argentina. Acta Hydrobiologica, 40: 161-172.

BAZÁN, G. I., S. B. ÁLVAREZ , M. C. MARTÍN \& J. BERNARDOS. 2004. Análisis de la taxocenosis de la ficoflora de la Laguna El Ojo de Agua, Uriburu, La Pampa, Argentina Biología Acuática, 20: 1-5.

BOLTOVSKOY, A., A. DIPPOLITO, M. FOGGETTA, N. GÓMEZ \& G. ÁLVAREZ. 1990. La Laguna Lobos y su afluente: Limnología descriptiva con especial referencia al plancton. Biología Acuática, 14. $37 \mathrm{pp}$.

CABRERA, A. L. \& A. WILLINK. 1980. Biogeografía de América Latina. Sec. Gral de la OEA, Washington, D.C. 271 pp.

CARBALlO, O. C., A. P. CALMELS, E. S. SOTORRES, J. L. MARANI \& J. A. SBROCCO. 2000. Evolución ambiental del Humedal Don Tomás, provincia de La Pampa, Argentina. V Seminario Latinoamericano de Calidad de Vida Urbana, Actas, Universidad de Bío-Bío, Chillán, Chile. CD 7 pp.

GARCÍA DE EMILIANI, M. O. \& P. J. DEPETRIS. 1982. A principal components analysis of the phytoplankton from a pond in the Paraná River River Valley, Argentina. Hidrobiologia, 89: 117-122.

GEITLER, L. 1932. Cyanophycae. Rabenhorst's Kryptogamen Flora 14, Akademische Verlagsgesellschaft. Leipzig. 1196 pp.

IZAGUIRRE, I. \& A. VINOCUR. 1994. Algal assemblages from shallow lakes of the Salado River Basin (Argentina). Hydrobiologia, 289: 57-64.

KOMÁREK, J. \& K. ANAGNOSTIDIS. 1989. Modern approach to the classification system of Cyanophytes, 4-Nostocales. Arch. Hydrobiol. Suppl. 82(1). Algol. Stud., 56: 247-345.

KOMÁREK, J. \& K. ANAGNOSTIDIS. 1999. Cyanoprokaryota. 1. Teil: Chroococcales. Süsswasserflora von Mitteleuropa. begr. von Pascher Hrsg. von H. Ettl. Jena. G. Fischer Bd. 19. 548 pp. 643 figures.

KOMÁREK, J. \& K. ANAGNOSTIDIS. 2005. Cyanoprokaryota. 1. Teil: $2^{\text {nd }}$ Part: Oscillatoriales. Süsswasserflora von Mitteleuropa. begr. von Pascher Hrsg. von H. Ettl. Jena. G. Fischer Bd. 19. 759 pp. 1010 figures.

KOMÁREK, J. \& B. FOTT. 1983. Systematik und Biologie. Chlorophyceae (Grünalgen) Ordnung: Chlorococcales. Die Binnengewässer. Das Phytoplankton des Süsswasser. von Huber-Pestalozzi. E. 
Schweizerbart'sche Verlagsbuchhandlung (Nägele u. Obermiller) Sttugart. Germany. 1043 pp.

LAWS, E. A. 1993. Aquatic Pollution. An Introductory Text. $2^{\circ}$ Ed. John Wiley \& Sons, INC. 611 pp.

LITCHMAN, E. 2000. Growthrates of phytoplankton under fluctuating light. Freshwat. Biol., 44: 223-235.

MAIDANA N. I. \& O. E. ROMERO. 1995. Diatoms from the hypersaline "La Amarga" Lake (La Pampa, Argentina). Cryptogamie. Algol., 16(3): 173188.

MARSHALL, H. G., L. BURCHARDT, T. A. EGERTON , K. STEFANIAK \& M. LANE. 2008. Potentially toxic cyanobacteria en Chesapeake Bay estuaries and a Virginia lake. In: Cyanobacterial Harmful algal blooms. State of the Science and Research Needs. H. Kenneth Hudnell (ed.): 172-173. Springer. USA.

PRESCOTT, G. W. 1951. Algae if the Western Great Lakes Area. Cranbrook Inst. Sci. Bull. 31. Bloomfield Hills, Michigan. 946 pp.

QUIRÓS R., C. R. M. BAIGUM, S. CUCH, R. DELFINO \& A. De NICHILO. 1988). Evaluación del rendimiento pesquero potencial de la Repúblico Argentina. I. Datos 1. Informe técnico $N^{o} 7$, Dpto. De Aguas Continentales, Instituto Nacional de Investigación y Desarrollo Pesquero. Mar del Plata. Argentina. $55 \mathrm{pp}$.

QUIRÓS, R., A. M. RENNELLA, M. B. BOVERI, J. J. ROSSO \& A. SOSNOVSKY. 2002. Factores que afectan la estructura y el funcionamiento de las lagunas pampeanas. Ecología Austral, 12: 175-185.
R Development Core Team.2009. R: A language and environment for statistical computing. R Foundation for Statistical Computing, Vienna, Austria. URL http://www.R-project.org.

RAI, H. \& G. HILL. 1980. Classification of Central Amazon Lakes on the basis of their microbiological and physico-chemical characteristics. Hydrobiologia, 72: 85-99.

RICHARDSON, T. L., C. E. GIBSON \& S. I. HEANEY. 2000. Temperature, growth and seasonal succession of phytoplanctonic Lake Baikal, Siberia. Freshwat. Biol., 44: 430-440.

ROMERO, O. E. 1993. Diatomeas (Bacillariophyceae) de la Provincia de La Pampa (Argentina) I. Darwiniana, 32(1-4): 303-313.

ROMERO, O. E. 1995. Diatomeas (Bacillariophyceae) de la Provincia de La Pampa (Argentina) II. Darwiniana, 33(1-4): 177-193.

SCHUELER, T. \& J. SIMPSON. 2003. Why urban lakes are different. Urban lake management. Watershed Protection Techniques, 1(2): 747-750.

VILA, I. \& R. PARDO. 2003. Respuesta de la estructura fitoplanctónica a las perturbaciones antrópicas en un lago templado. Limnetica, 22(1-2): 93-102.

WENZEL, M. T., S. B. ALVAREZ \& G. I. BAZÁN, 1996. Estudio preliminar de las cianofíceas del Embalse Casa de Piedra (Pcia. de La Pampa). Argentina. Physis. Secc. B., 51(120-121): 9-16.

ZOLACAR DE DOMITROVIC, Y., C. A. BONETTO \& H. G. LANCELLE, 1982. Algunos aspectos limnológicos de la laguna Herradura (Formosa, Argentina). Ecosur, 9: 171-188. 